\title{
Avoidance and Early Diagnosis of Erroneous Permanent Pacemaker Placement into the Left Ventricle: A Case Report
}

\author{
Abdelrahman Osman ${ }^{1}$ and Ali Ahmad ${ }^{2}$ \\ ${ }^{1}$ The Ohio State University College of Arts and Sciences \\ ${ }^{2}$ Affiliation not available
}

March 9, 2021

\begin{abstract}
Pacemakers are implanted through the subclavian vein to the right side of the heart. In rare cases, pacemakers may be inserted into the left ventricle via the subclavian artery, carrying a risk of thromboembolism. This is a case of a pacemaker inserted via the subclavian artery into the left ventricle.
\end{abstract}

\section{Introduction}

Implantation of pacemakers has increased in recent decades and has become an important tool to treat bradycardia. With access commonly through the left subclavian or cephalic vein, pacemaker leads are positioned into alternative pacing sites where they can stimulate the heart to restore normal rhythm (1). Pacemaker lead insertion through the left subclavian artery going back to the aortic valve into the left ventricular cavity is an extremely rare iatrogenic complication. The frequency of this complication is unknown but believed to be largely unreported. Common complications resulting from entry through the subclavian artery include pneumothorax and thromboembolic events (2).

Because of these complications, immediate identification and treatment is required before the patient leaves the electrophysiology lab, usually by using fluoroscopy, pacemaker interrogation, or a 12-lead electrocardiogram (ECG). If left undiagnosed, improperly placed leads can lead to serious systemic thromboembolism. Surgical extraction of an arterial lead can also lead to major life-threatening vascular complication (3). We report a case of a 59-year-old man who inadvertently had a pacemaker lead implanted via the subclavian artery.

\section{Case Report}

A 59-year-old man with history of chronic obstructive pulmonary disease presented to the emergency room with severe sinus bradycardia and hypotension. He was diaphoretic and denied any chest pain. He gave a history of recurrent dizziness and near syncopal episodes. The patient was not on any atrioventricular nodal blocking agents and initial blood work showed no reversible cause of his severe sinus bradycardia.

The patient was started on intravenous fluid hydration and a bedside echocardiogram was obtained. Echocardiogram showed normal biventricular systolic function and no significant valvular abnormalities. Atropine was given but resulted in minimal, transient improvement in his heart rate to the low 50 s. The systolic blood pressure remained below $80 \mathrm{mmHg}$. Dopamine followed by norepinephrine drips were administered and the patient was sent to the electrophysiology lab for dual chamber permanent pacemaker therapy.

During implantation of a permanent pacemaker, the right atrial lead was appropriately placed via the left subclavian vein into the right atrial appendage. However, the ventricular lead was placed via the subclavian artery into the left ventricular cavity. He was admitted to the hospital overnight and a chest x-ray was 
obtained the next day, showing no evidence of pneumothorax (Figure 1 ). Surprisingly, his ECG showed atrial pacing with long AV delay and intermittent ventricular pacing with the paced ventricular beats showing right bundle branch block morphology contrary to the expected left bundle branch block morphology seen when the lead is inside the right ventricular cavity (Figure 2 ). A limited bedside Echo was obtained that showed the ventricular lead clearly crossing the aortic valve to the left ventricular cavity and fixed into the inferolateral wall of the left ventricle (Figure 3 ). Early recognition of this serious pacemaker implantation complication led to pacemaker revision in the same day with extraction of the arterial lead and reinsertion of the ventricular lead via the left subclavian vein into the right ventricular cavity (Figure 4 ). The pacemaker revision went uncomplicated.

\section{Discussion}

Cardiac pacemakers and defibrillators are cornerstones in the management of patients with heart rate and/or rhythm problems. There is a steady growth in the use of pacemaker and defibrillator therapy in the United States and across the world (1-3). Although commonly inserted with access through the left subclavian or cephalic vein, inadvertent entry through the subclavian artery can lead to iatrogenic complications, including thromboembolism. Quick diagnosis of this event is essential, as leaving the lead is associated with an increased risk of life-threatening thromboembolism. Thrombus formation on the lead can occur within days, and fibrous tissue may begin developing in just a few months (4).

There are several methods available to avoid the occurrence of such events. When subclavian vein puncture is attempted, the subclavian artery may also be punctured. Arteriography may be used to identify the accessed vessel. In addition, successful venipuncture may be observed as the guidewire advances into the inferior vena cava under fluoroscopy. If there are ventricular premature beats with right bundle branch block pattern soon after the guidewire enters the ventricular cavity, the possibility of the guidewire entering through the artery must be considered. After the pacemaker is implanted, correct positioning of the pacemaker may also be seen through ECG. Right ventricular pacing is indicated when ECG shows left bundle branch block appearance, while left ventricular pacing is seen in right bundle branch block patterns (5).

Removal of the lead is one solution to avoid thromboembolic events but comes with other complications. Extraction of transarterially placed leads is associated with high thromboembolic risk as well as risk of bleeding from the arterial entry site. Furthermore, lead removal can lead to ventricular perforation or cardiac tamponade, and may cause trauma to the aorta, aortic valve, and coronary arteries (6). Given the direct connection to systemic circulation, any lead manipulation can potentially lead to systemic embolization. Due to the unnecessary risks pertaining to surgical removal, conservative management with lifelong anticoagulation may be an acceptable alternative in chronically implanted left ventricular cavity leads.

\section{Conclusion}

Insertion of a pacemaker lead through the subclavian artery is extremely rare and believed to be markedly underreported. Adverse events resulting from this iatrogenic complication includes pneumothorax, arterial injury and thromboembolic events. Immediate identification and diagnosis of this complication is essential. Careful interpretation of post-implantation ECG lead is of extreme value to identify this complication. Proper utilization of imaging modalities including lateral chest x-ray view and echocardiography should be adopted whenever improper lead placement is suspected. 


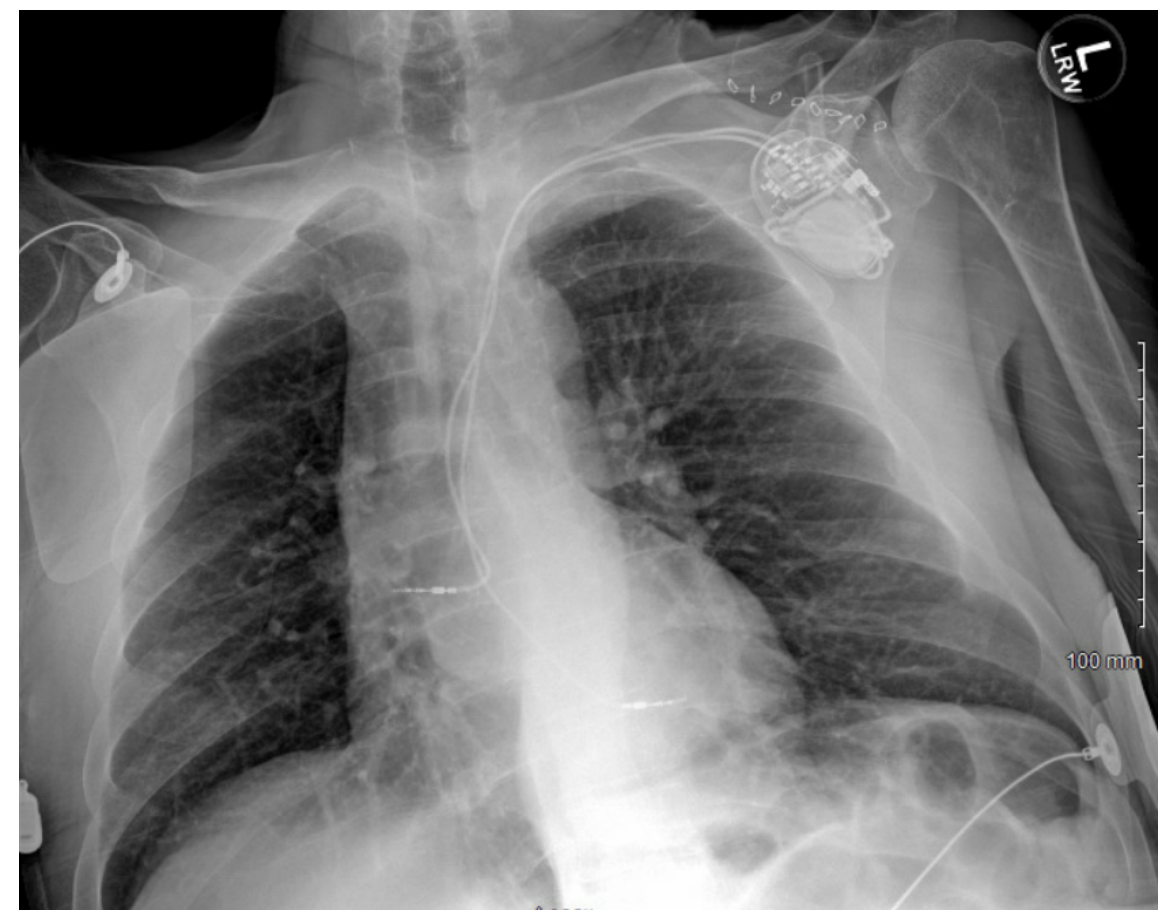

Figure 1. Anteroposterior view of the chest showing no evidence of pneumothorax with the atrial lead in good position while the ventricular lead is higher. This can suggest inadvertent lead placement but lateral chest $\mathrm{x}$-ray is needed for further evaluation.

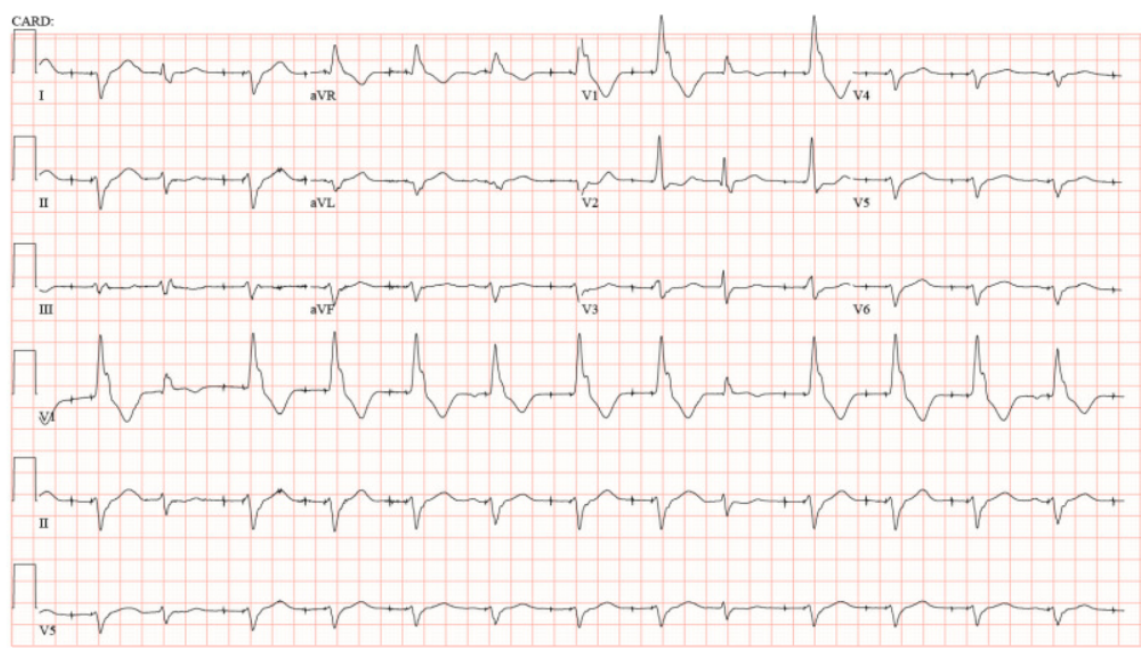

Figue 2. A 12-lead ECG showing atrial pacing with long AV delay (blue circle) and intermittent ventricular pacing (red circle). The key point is that the ventricular paced beats are conducted with right bundle branch block morphology suggesting that the ventricular lead is located in the left ventricle. 


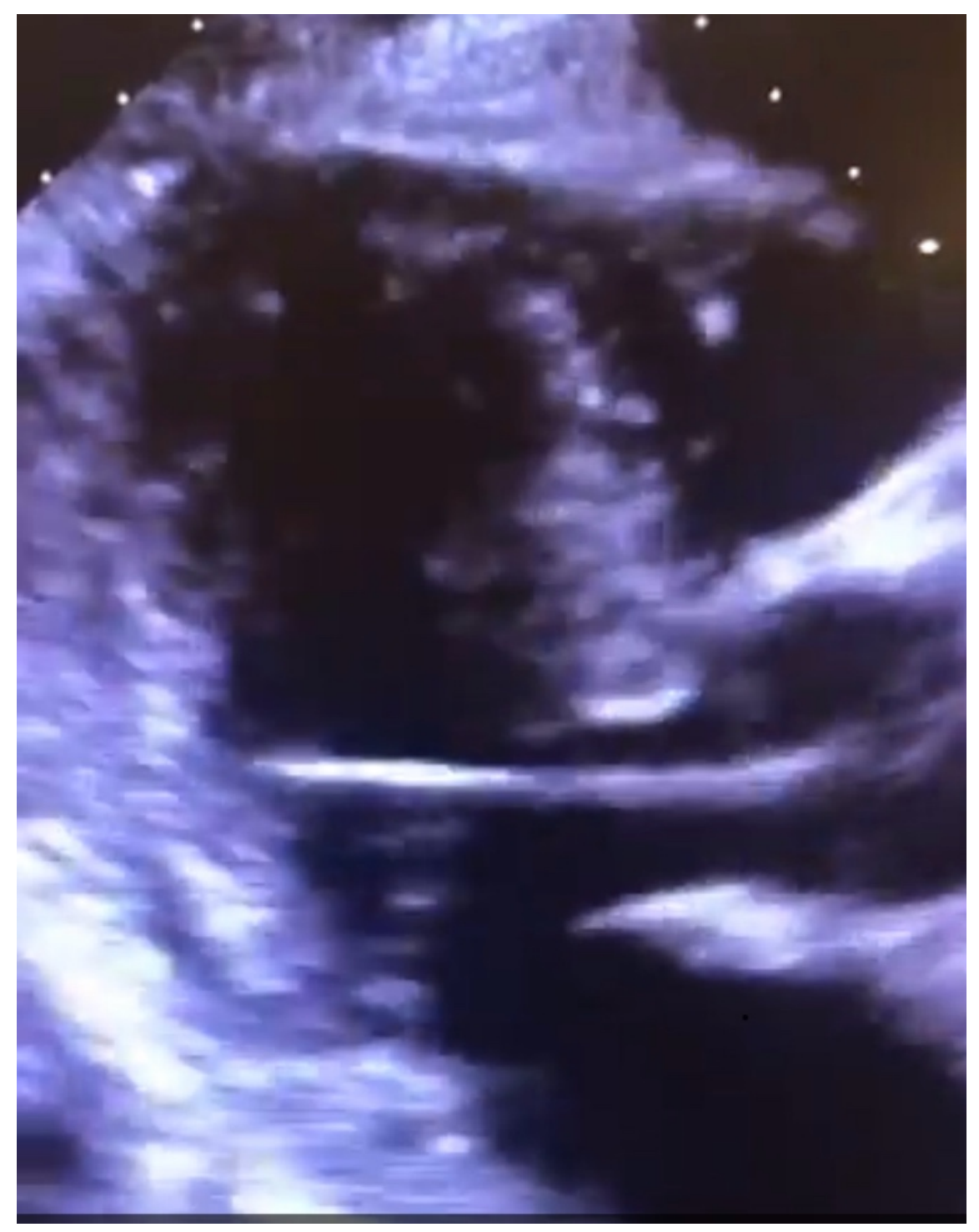

Figure 3 Parasternal long axis view showing pacemaker lead (arrow) crossing the aortic valve into the left ventricular cavity and fixed to the inferolateral wall of the left ventricle. 


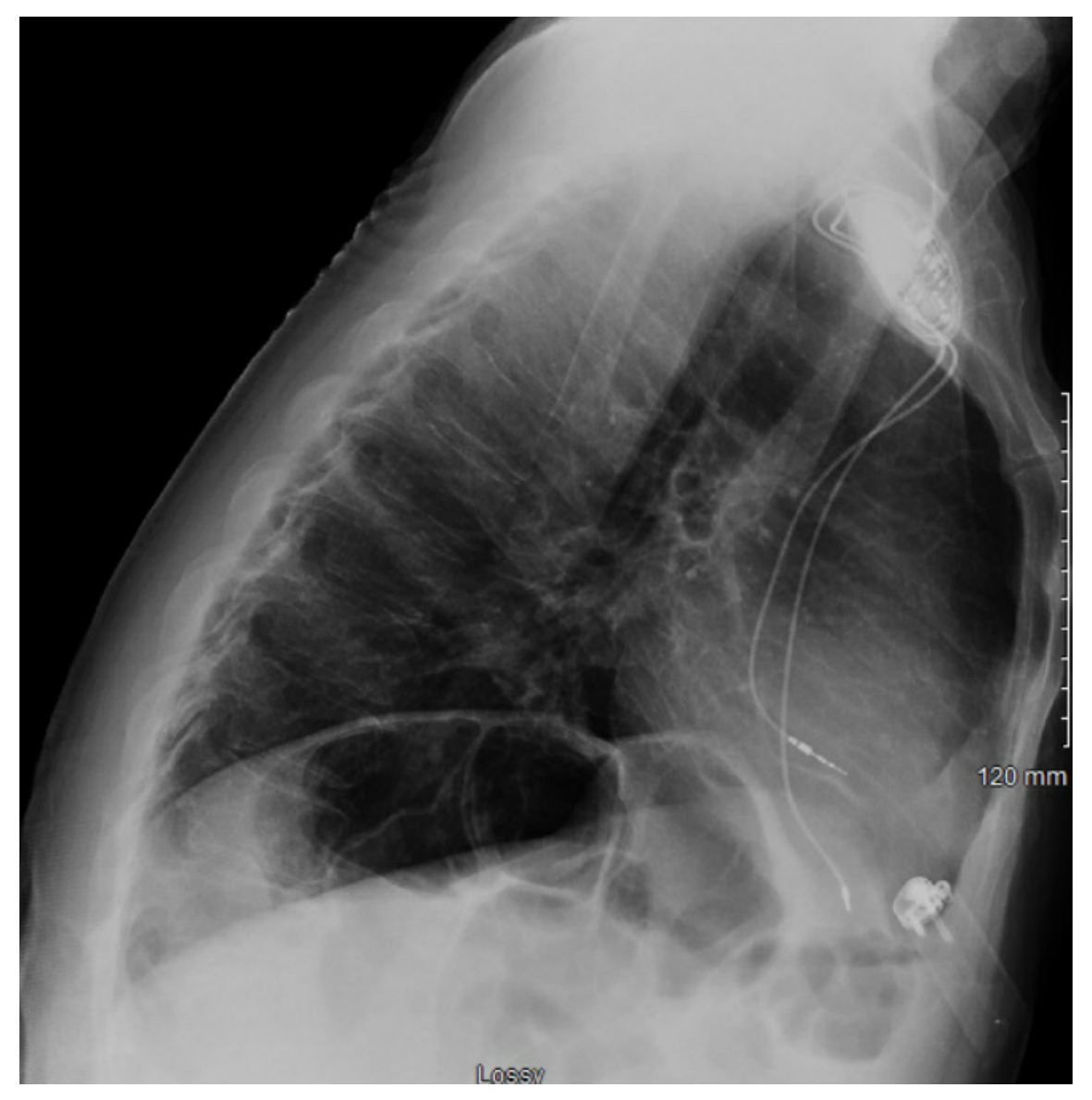

Figure 4. Lateral chest x-ray view post-pacemaker revision with the ventricular lead directed anteriorly suggesting right ventricular position.

\section{References}

1. Arnold j.Greenspon, Jasmine D. Patel, Edmund Lau, Jorge A. Ochoa, Daniel R. Frisch, Reginald T. Ho, Behazd B. Pavri, and Steven M Kurtz. Trends in Permanent Pacemaker Implantation in the United States from 1993 to 2009: Increasing Complexity of the Patients and Procedures. JACC. 2012 Oct, 60 (16) 1540-1545.

2. Harry G. Mond M.D., Marleen Erwin R.R.T/C., Hugo Ector M.D., Alessandro Proclemer M.D., The World Survey of Cardiac Pacing and Cardioverter-Defibrillators: Calendar Year 2005 An International Cardiac Pacing and Electrophysiology Society (ICPES) project. Pacing Clin Electrophysiol 2008:31:1202

3. Daniel Z. Uslan, Imad M Tleyjeh, Larry M Baddour, Paul A Friedman, Sarah M Jenkins: Temporal Trends in Permanent Pacemaker Implanatoion: A Population-based Study. AHJ 2008; 155 (5), 896903.

4. Tarek Ajam, MD. Cardioverter-Defibrillator Implantation Technique: Approach Considerations, Incision, Creation of Pocket, Medscape, 5 Dec. 2020, emedicine.medscape.com/article/1839525-technique.

5. Tang G., Zhai C., Wang Z., et al. Permanent pacemaker implanted into patient's left ventricle via subclavian artery by mistake: a case report. BMC Cardiovasc Disorders 2015;15:39.

6. Meyhoefer J., Lehmann H., Minden H., et al. Closure of the subclavian artery puncture site with a percutaneous suture device after removal of an arterial pacemaker lead. Europace 2006;8 :1070-2.

7. Dissman R, Wolthoff U, Zabel M. Double left ventricular pacing following accidental malpositioning 
of the right ventricular electrode during implantation of a cardiac resynchronization therapy device. Journal of Cardiothoracic Surgery 2013;8 :162

8. Kosmidou I., Karmpaliotis D., Kandzari D., et al. Inadvertent transarterial lead placement in the left ventricle and aortic cusp: percutaneous lead removal with carotid embolic protection and stent graft placement. Indian Pacing and Electrophysiol J2012;12:269-273.

9. Love C.J., Wilkoff B.L., Byrd C.L., et al. Recommendations for extraction of chronically implanted transvenous pacing and defibrillator leads: indications, facilities, training. Pacing Clin Electrophysiol $2000 ; 23: 544-551$. 